\title{
Author Correction: Immune cell profiling of COVID-19 patients in the recovery stage by single-cell sequencing
}

Wen Wen, Wenru Su, Hao Tang, Wenqing Le, Xiaopeng Zhang, Yingfeng Zheng, Xiuxing Liu, Lihui Xie, Jianmin Li, Jinguo Ye, Liwei Dong, Xiuliang Cui, Yushan Miao, Depeng Wang, Jiantao Dong, Chuanle Xiao, Wei Chen and Hongyang Wang

Correction to: Cell Discovery (2020) 6:31 https://doi.org/10.1038/s41421-020-0168-9

Published online 04 May 2020

Following publication of the original article ${ }^{1}$, an error was identified in the Data availability section. The accession number of the dataset was updated. The corrected Data availability appears below:

The raw sequence data reported in this paper has been deposited in the Genome Sequence Archive (Genomics, Proteomics \& Bioinformatics 2017) in National Genomics Data Center (Nucleic Acids Res 2020), Beijing Institute of
Genomics (China National Center for Bioinformation), Chinese Academy of Sciences, under Project Accession No. PRJCA002413 (GSA Accession No. CRA002497) that are publicly accessible at https://bigd.big.ac.cn/gsa.

Published online: 20 June 2020

\footnotetext{
Reference

1. Wen, W., Su, W., Tang, H. et al. Immune cell profiling of COVID-19 patients in the recovery stage by single-cell sequencing. Cell Discov. 6, 31 (2020).
} 